\title{
Signal Enhancement of Abiotically-Synthesized RNA Oligonucleotides and other Biopolymers using Unmodified Fused Silica in MALDI-MS
}

\author{
Lauren M. Cassidy, Yingying Dong, Prakash C. Joshi, Michael F. Aldersley, \\ James P. Ferris, Linda B. McGown
}

Department of Chemistry and Chemical Biology The New York Center for Astrobiology, Rensselaer Polytechnic Institute, Troy, NY, USA

\begin{abstract}
Metal is the standard desorption platform for MALDI-MS but other surfaces have been shown to offer advantages for particular types of analytes or applications. One such substrate is fused silica, which has been employed for matrix-free detection of low mass analytes and for affinity MALDI-MS in which binding ligands are immobilized at the fused silica surface. The present work reports improved MALDI-MS detection of RNA oligonucleotides, including polyA, polyU, and polyA/U, at the high end of the mass range when unmodified fused silica is used instead of stainless steel as the MALDI target. The RNA oligonucleotides were abiotically synthesized from activated monomers on catalytic clay surfaces. Further investigation found enhanced signals as well for other anionic biopolymers, including DNA oligonucleotides and heparin. Enhancement also was observed for dextran, which is neutral, indicating that the effect is not restricted to anionic biopolymers. Among more general analytical applications, the results are particularly relevant to rapid screening of abiotic RNA polymerization toward elucidating pathways to life on Earth.
\end{abstract}

Key words: MALDI-MS, RNA world, RNA, DNA, Heparin, Dextran, Prebiotic synthesis, Fused silica target, Biopolymers

\section{Introduction}

$\mathrm{M}$ atrix-assisted laser desorption ionization mass spectrometry (MALDI-MS) is widely used for mass analysis of peptides and proteins. It is also useful for other biological and synthetic polymers but often with lower detectability. In particular, difficulties associated with detection of nucleic acids and optimization of experimental methods have been discussed in the literature $[1,2]$. Based primarily on the extensive body of work on peptides and

Electronic supplementary material The online version of this article (doi:10.1007/s13361-011-0113-0) contains supplementary material, which is available to authorized users.

Correspondence to: Linda B. McGown; e-mail: mcgowl@rpi.edu proteins for which conductive surfaces provide optimal detection, it is generally accepted practice to use metals, such as stainless steel or aluminum, as the MALDI target for all analytes. We were therefore surprised to discover in the present work that MALDI-MS detection of RNA oligonucleotides can be extended to higher mass ranges by using unmodified fused silica instead of metal targets. Activated fused silica or other target materials, such as carbon, metal oxides, or nanoparticles, have been used in matrix-free techniques to improve detection or eliminate matrix interference in detection of low molecular weight analytes [3-10], but improved detection of polymers at the higher end of the molecular weight range is unexpected.

The motivation for this work is the study of abiotic synthesis of RNA on montmorillonite clay surfaces. These clays belong to the class A phyllosilicate clays known as 
smectite or "swelling" clays. They can cause polymerization of nucleoside 5'- imidazoles (RNA monomers, ImpN, where $\mathrm{N}=\mathrm{A}, \mathrm{U}, \mathrm{C}, \mathrm{G})$ under mildly acidic $(\mathrm{pH}$ 6-8) conditions $[11,12]$, and are of interest in the "RNA world" hypothesis that supposes RNA was the primary biomolecule of life, regardless of whether it was terrestrial or extraterrestrial in origin [13].

In order to determine if the enhancement is a general effect for anionic biopolymers or specific to the RNA oligonucleotides, we tested DNA and heparin. For comparison, we also tested the neutral biopolymer dextran.

\section{Experimental}

\section{Materials}

Fused silica plates were from Valley Design (Shirley, MA, USA). Sinapic acid (SA), 2,4,6-trihydroxyacetophenone (THAP), $\alpha$-cyano-4-hydroxycinnamic acid (CHCA), 2-(4-hydroxyphenylazo) benzoic acid (HABA), dextran ( $\sim 4-76 \mathrm{kDa}$, from Leuconostoc mesenteroides), adenosine 5'-monophosphate (AMP), and uridine monophosphate (UMP) were from Sigma (St. Louis, MO, USA). Montmorillonite was a gift from The American Colloid Company (Arlington Heights, IL, USA). Heparin ( 14 kDa) was a gift from Dr. Robert J. Linhardt at Rensselaer Polytechnic Institute. A DNA standard ladder was custom synthesized by BioVentures (Murfreesboro, TN, USA). The ladder contains five dsDNA fragments $(1 \mathrm{pmol} / \mathrm{band} / \mu \mathrm{L})$ that are $50,75,100,125$, and $150 \mathrm{bp}$ in length. Deionized distilled water was used for all sample preparations and experiments.

\section{ImpN Polymerization}

Monomeric AMP and UMP were activated by adding imidazole to the $5^{\prime}$-phosphate as described in the literature $[11,12]$. Once activated, the imidazole monophosphate adenosine (ImpA) or uridine (ImpU) was dissolved in RNase free water to a concentration of $15 \mathrm{mM}$. Homoionic montmorillonite (containing sodium) was prepared by the procedure of Banin et al. [14]. The ImpN solution was added to lyophilized montmorillonite clay and vortexed to form a homogenous mixture. After $2 \mathrm{~d}$ of agitation, the sample was centrifuged, and the supernatant collected. In order to ensure maximum product recovery, clays were resuspended in $20 \mu \mathrm{L}$ of ACN $(20 \% \mathrm{vol} / \mathrm{vol})$ in RNase free water, agitated until homogeneous, and centrifuged. The supernatant from this second extraction was pooled with the supernatant from the first extraction.

Verification of the polymerization reaction was accomplished using ion exchange HPLC. This is shown in Figure S1 and Table S1 (see Supplementary Material) for
ImpA, for which oligonucleotides as long as 8-mers were detected.

\section{MALDI-TOF-MS Analysis}

MALDI-TOF-MS was performed using a Bruker Autoflex II instrument (Bruker Daltonics, Billerica, MA, USA). The targets were MTP 384 massive stainless steel targets that were either unmodified or modified to accommodate a $5 \times 5 \mathrm{~cm}$ fused silica plate. MALDI matrices including SA, THAP, CHCA, and HABA were compared for each sample. CHCA provided best detection for all samples except dextran, for which HABA provided the best results.

All analyses were performed in the negative ion mode, except for heparin, which was analyzed in both positive and negative ion modes. The RNA oligomer products were analyzed in reflectron mode with the following settings: ion source $1,19.00 \mathrm{kV}$; ion source $2,16.85 \mathrm{kV}$; lens, $8.5 \mathrm{kV}$; reflector, $20.0 \mathrm{kV}$; system energy, $115.7 \pm 5.0 \mu \mathrm{J}$; pulsed ion ext., $50 \mathrm{~ns}$. All other samples were run in linear mode with the following settings: ion source $1,19.00 \mathrm{kV}$; ion source 2, $18.50 \mathrm{kV}$; lens, $8.4 \mathrm{kV}$; system energy, $115.7 \pm 5.0 \mu \mathrm{J}$; pulsed ion ext., $500 \mathrm{~ns}$. In all cases, relative laser power for desorption was $31 \%-41 \%$ for the fused silica target and $21 \%-31 \%$ for the stainless steel target.

For all samples, $1 \mu \mathrm{L}$ of sample solution was mixed with $2 \mu \mathrm{L}$ matrix, deposited on the target and allowed to fully crystallize. For the RNA samples, there were 70 shots per location for 64 locations over six spots on silica and 61 locations over seven spots on stainless steel. For the DNA ladder, there were 100 shots per location for four locations on one spot on silica and three locations for one spot on stainless steel. For heparin, there were 70 shots per location for six locations/spot for two spots on silica and for a total of 28 locations over three spots on stainless steel. For dextran, there were 70 shots per location for four locations on one spot on silica and for 20 locations on one spot on stainless steel. The shots were summed and smoothed using the Savitsky Golay polynomial regression algorithm with a width of $0.2 \mathrm{~m} / z$. Baseline subtraction was not used. Spectra show the best results for a sample on each type of surface. Tabulated results were calculated using values for peaks with $\mathrm{S} / \mathrm{N} \geq 3$. The $\mathrm{S} / \mathrm{N}$ value is calculated by the instrument software as the height of the peak above the baseline divided by the standard deviation of the baseline noise.

The extract containing RNA products was analyzed directly without further treatment. Assuming 100\% extraction efficiency of the products, $1 \mu \mathrm{L}$ of the extract is estimated to contain $\sim 5$ nmol total RNA product, at least a third of which is monomer according to the HPLC analysis (see Supplementary Material). The DNA fragments $(1 \mathrm{pmol} / \mathrm{band} / \mu \mathrm{L})$ were heat-denatured prior to analysis but without any other treatment or dilution. Heparin was prepared by dissolution of a lyophilized sample in water at a concentration of $1 \mathrm{mg} / \mathrm{mL}$, followed by 10 -fold dilution in water. Dextran was prepared by dissolution in water at a concentration of $10 \mu \mathrm{g} / \mathrm{mL}$. 

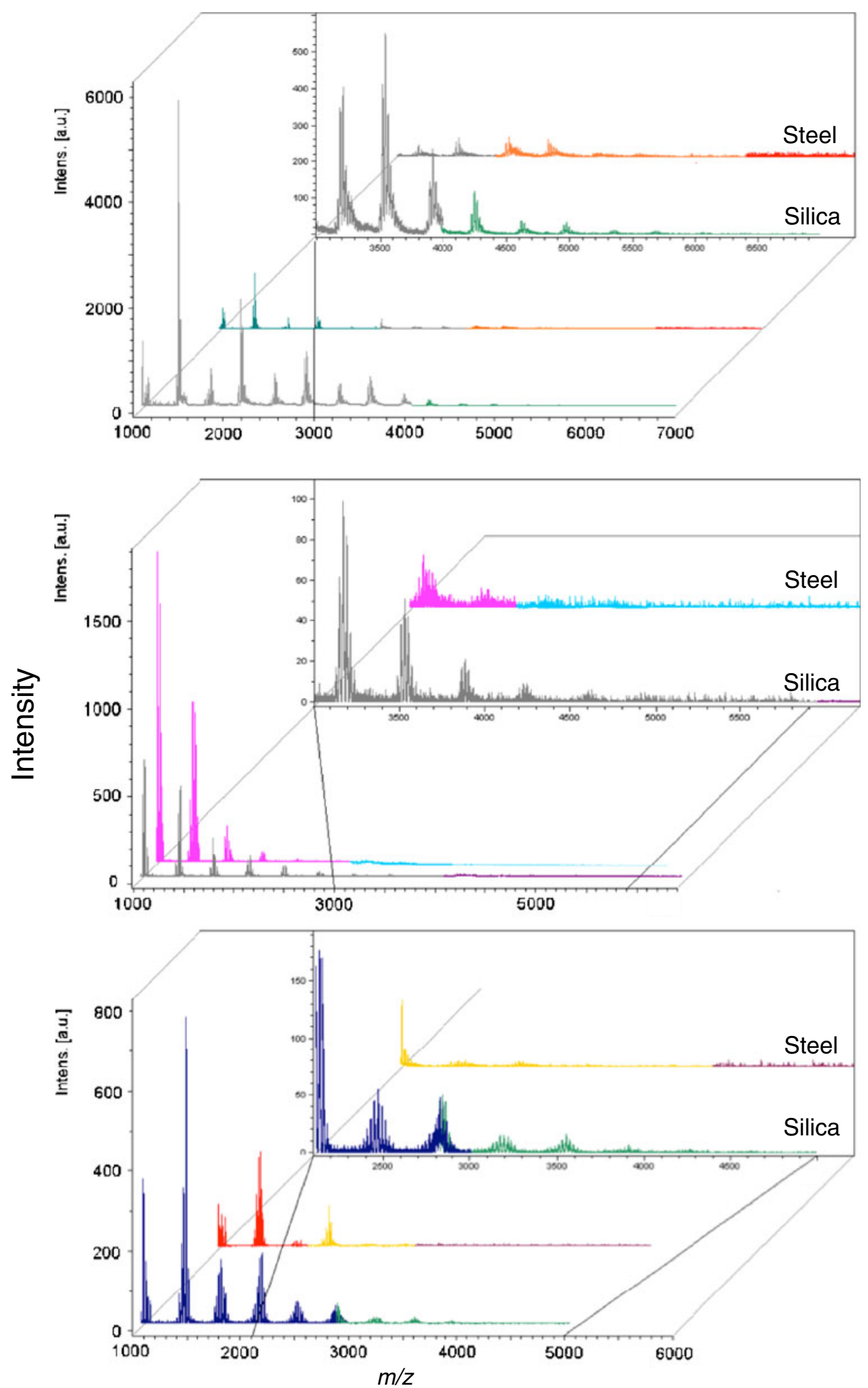

Figure 1. MALDI-MS spectra of ImpA (top), ImpU (middle), and ImpA/U (bottom) products using THAP matrix in negative ion, reflectron mode. Foreground: desorbed from fused silica. Background: desorbed from stainless steel. Inset (upper right) in each figure shows higher $\mathrm{m} / \mathrm{z}$ portion of spectra on expanded y-axis. Different colors indicate spectra that were acquired for different $\mathrm{m} / \mathrm{z}$ ranges that were then concatenated in the figure to show full range. The first peak at $\sim \mathrm{m} / \mathrm{z} 1000$ corresponds to the 3-mer. Successive peaks, spaced by $\sim 300 \mathrm{~m} / \mathrm{z}$ units, correspond to the 4-mer, 5-mer, etc 


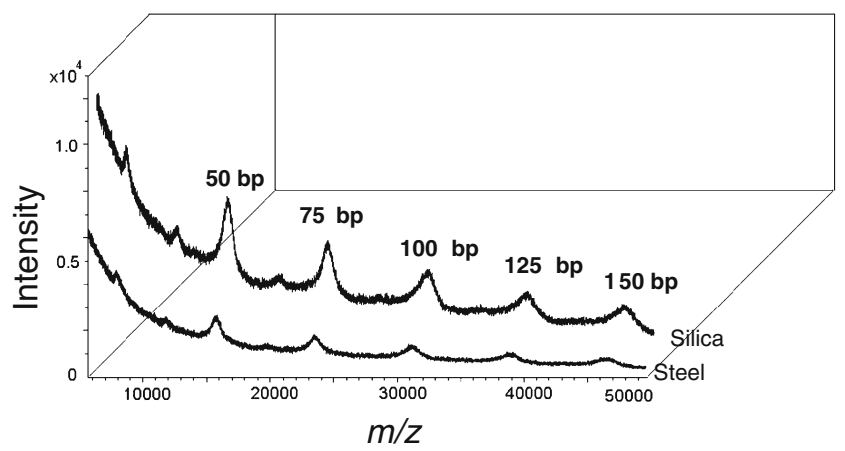

Figure 2. MALDI-MS spectra of five denatured dsDNA fragments $(50,75,100,125,150 \mathrm{bp})$, acquired using THAP matrix in negative ion, linear mode. Foreground: desorbed from steel. Background: desorbed from silica

\section{Results}

\section{RNA Oligonucleotides}

Figure 1 shows representative mass spectra for the ImpA, ImpU, and ImpA/U products (3-mer and above) analyzed on stainless steel and fused silica targets. The spectra are composites of spectra acquired over different $\mathrm{m} / \mathrm{z}$ ranges (represented by different colors) that were collected to maximize detection of peaks. Instrumental conditions and MALDI matrix were optimized for the samples on each of the surfaces. The matrix CHCA provided the best results. Table S2 shows the mean signal-to-noise ratios $(\mathrm{S} / \mathrm{N})$, peak intensities and associated standard deviations (when possible) for the oligomers (2-mer and above) averaged for samples on different spots and, when applicable, for overlapping regions of spectra taken for different $\mathrm{m} / \mathrm{z}$ ranges on a given spot. Only those peaks with $\mathrm{S} / \mathrm{N} \geq 3$ were included.

The results show that detection is extended from 15-mers to 18-mers for PolyA, from 11-mers to 13-mers for PolyU, and from 8- to 12-mers for PolyA/U when fused silica is used instead of stainless steel. It is important to note that the relative success in detecting a particular oligomer is a function both of the ability of MALDI to detect that oligomer and the relative abundance of that oligomer in the extracted products.

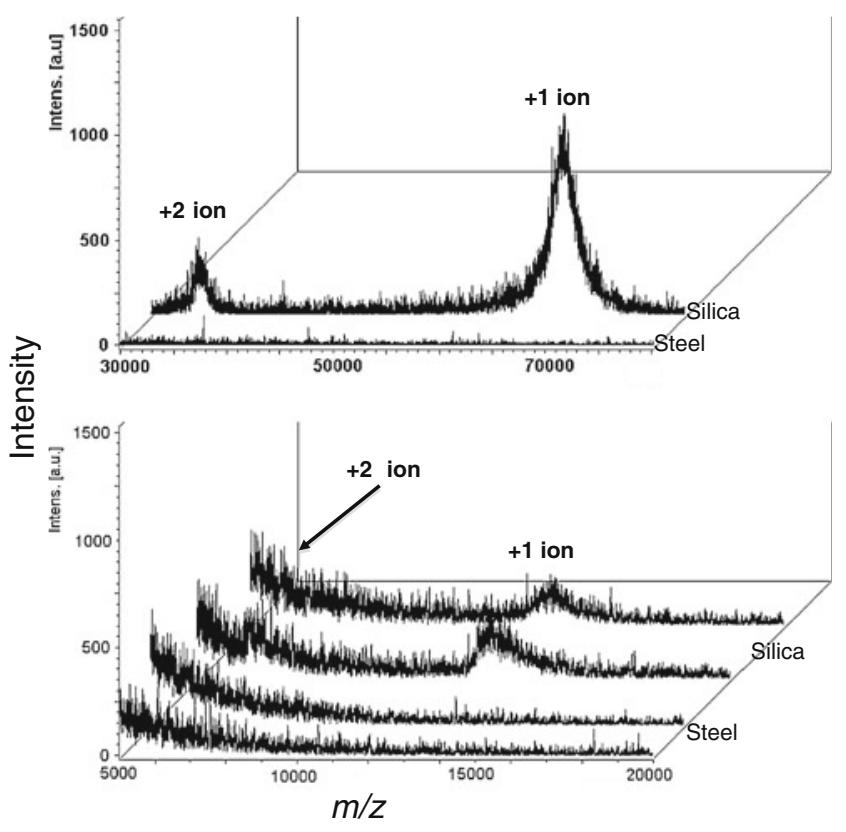

Figure 3. Top: MALDI-MS spectra of dextran polymers (average MW 64-70 kDa) acquired using CHCA matrix in negative ion, linear mode. Foreground: desorbed from steel. Background: desorbed from silica. Bottom: MALDI-MS spectra of heparin (MW 14 kDa) acquired using HABA matrix in linear mode. Spectra from foreground to background: desorbed from steel in positive ion mode; desorbed from steel in negative ion mode; desorbed from silica in positive ion mode; desorbed from silica in negative ion mode

\section{DNA Oligonucleotides}

Figure 2 shows a comparison of the spectra obtained for the DNA ladder on fused silica versus stainless steel. As for the RNA oligomers, CHCA matrix provided the best results, and detectability was better on fused silica than on steel regardless of matrix. Table 1 shows the mean $\mathrm{S} / \mathrm{N}$ and relative standard deviations of the five oligomer peaks in the spectra calculated from $n$ different locations on a sample spot on each surface. For all five peaks, the mean $\mathrm{S} / \mathrm{N}$ on silica is twice that on steel, and the mean relative standard deviation of the $\mathrm{S} / \mathrm{N}$ is in the range of $40 \%-43 \%$ for silica and $63 \%-70 \%$ for steel. These results indicate that the trend for DNA is the same as that for RNA.

Table 1. MALDI-MS Detectability of 5 DNA Ladder Peaks on Fused Silica and on Stainless Steel, Expressed as Mean S/N and Relative Standard Deviation (RSD) for Spectra Acquired at $n$ Locations on a Sample Spot on Each Surface

\begin{tabular}{lllll}
\hline Peak & Silica & $(n=4)$ & Steel & $(n=3)$ \\
\cline { 2 - 5 } & S/N & RSD (\%) & S/N & 143 \\
RSD (\%) \\
\hline $50 \mathrm{bp}$ & 271 & 41 & 98 & 63 \\
$75 \mathrm{bp}$ & 205 & 40 & 72 & 65 \\
$100 \mathrm{bp}$ & 157 & 42 & 57 & 69 \\
$125 \mathrm{bp}$ & 122 & 43 & 46 & 67 \\
$150 \mathrm{bp}$ & 104 & 42 & & \\
\hline
\end{tabular}




\section{Heparin and Dextran}

In order to determine if the enhancement observed at higher $\mathrm{m} / \mathrm{z}$ at fused silica surfaces is unique to the oligonucleotides or a more general phenomenon for anionic biopolymers, MALDIMS was attempted for heparin. The matrix CHCA $(20 \mathrm{mg} / \mathrm{mL})$ saturated in water (50:50 matrix:water) provided the best results. The parent ion peak around $m / z 14 \mathrm{k}$ was detected on fused silica but could not be reproducibly detected on stainless steel (Fig. 3, top). The parent ion peak was not observed at either surface when other matrices were used. The peak was observed in both negative and positive ion modes (the latter used in an attempt to improve detectability), but with a much more precise $\mathrm{S} / \mathrm{N}$ in the former $(21 \pm 10(\mathrm{rsd}=48 \%)$ versus 165 $\pm 208(\mathrm{rsd}=126 \%)(n=7)$. The shape of the major peak was also different in the two modes, indicating differences between desorption as positive vs. negative ions.

We next investigated dextran to determine if the enhancement occurs for neutral biopolymers as well as anionic biopolymers. The matrix HABA provided the best results. Like heparin, the parent ion peak of dextran was detectable only on silica, not on steel (Fig. 3, bottom). On silica, the mean $\mathrm{S} / \mathrm{N}$ were $106 \pm 7(\mathrm{rsd}=7 \%)$ for the major peak at $\mathrm{m} / \mathrm{z}$ 69,400 and $43 \pm 7(\mathrm{rsd}=16 \%)$ for the +2 ion peak at $\mathrm{m} / \mathrm{z}$ $34,700(n=7)$. This result shows that the enhancement is not restricted to anionic biopolymers.

\section{Conclusions}

Extensive experience in our laboratory and across the community shows that cationic biopolymers, such as peptides and proteins, exhibit better MALDI-MS detectability on metallic or semiconductive surfaces compared with fused silica. The present work indicates that fused silica may provide better detection of anionic biopolymers at higher mass ranges when the laser power is optimized. This trend was observed regardless of the MALDI matrix used. The improved detection on fused silica was observed for the neutral biopolymer dextran as well. These results may provide new insight into MALDI mechanisms. The use of fused silica targets is a simple way to improve detectability that could be particularly relevant to rapid screening of RNA products from abiotic reactions such as those on catalytic clays. In this regard, it is worth noting that MALDI-MS of the RNA products using fused silica targets extended detectability beyond the 8-mer observed using HPLC (shown in the supplementary results) to longer strands (up to 18-mer for ImpA), which is important for rapid screening of abiotic RNA reaction products.

\section{Acknowledgment}

The authors acknowledge financial support for this research by the NASA Astrobiology Institute (grant NNA09DA80A).

\section{References}

1. Sauer, S.: The essence of DNA sample preparation for MALDI mass spectrometry. J. Biochem. Biophys. Methods 70, 311-318 (2007)

2. Sudha, R., Zenobi, R.: The detection and stability of DNA duplexes probed by MALDI mass spectrometry. Helvetica Chim. Acta 85, 31363143 (2002)

3. Peterson, D.S.: Matrix-free methods for laser desorption/ionization mass spectrometry. Mass Spectrom. Rev. 26, 19-34 (2007)

4. Cohen, L., Go, E.P., Siuzdak, G.: In: Hillenkamp, F., Peter-Katalinic, J. (eds.) MALDI-MS: A Practical Guide to Instrumentation, Methods and Applications, pp. 299-337. Wiley-VCH Verlag $\mathrm{GmbH}$ and Co, Weinheim (2007)

5. Guild, G.E., Lenehan, C.E., Walker, G.S.: Surface-assisted laser desorption ionization time-of-flight mass spectrometry with an activated carbon surface for the rapid detection of underivatized steroids. Int. J. Mass Spectrom. 294, 16-22 (2010)

6. Wei, J., Buriak, J.M., Siuzdak, G.: Desorption-ionization mass spectrometry on porous silicon. Nature 399, 243-246 (1999)

7. Cuiffi, J.D., Hayes, D.J., Fonash, S.J., Brown, K.N., Jones, A.D.: Desorption-ionization mass spectrometry using deposited nanostructured silicon films. Anal. Chem. 73, 1292-1295 (2001)

8. Liu, Q., Xiao, Y., Pagan-Miranda, C., Chiu, Y.M., He, L.: Metabolite imaging using matrix-enhanced surface-assisted laser desorption/ionization mass spectrometry (ME-SALDI-MS). J. Am. Soc. Mass Spectrom. 20, 80-88 (2009)

9. Liu, Q., Guo, Z., He, L.: Mass spectrometry imaging of small molecules using desorption/ionization on silicon. Anal. Chem. 79, 3535-3541 (2007)

10. Amini, N., Shariatgorji, M., Thorsén, G.: SALDI-MS signal enhancement using oxidized graphitized carbon black nanoparticles. J. Am. Soc. Mass Spectrom. 20, 1207-1213 (2009)

11. Ferris, J.P.: Mineral catalysis and prebiotic synthesis: montmorillonitecatalyzed formation of RNA. Elements 1, 145-149 (2005)

12. Ferris, J.P.: Montmorillonite-catalyzed formation of RNA oligomers: the possible role of catalysis in the origins of life. Philos. Trans. R. Soc. London, Ser. B 361, 1777-1786 (2006)

13. Simakov, M.B., Kuzicheva, E.A.: Abiotic photochemical synthesis on surface of meteorites and other small bodies. Adv. Space Res. 36, 190194 (2005)

14. Banin, A., Lawless, J.G., Mazzurco, J., Church, F.M., Margulies, L., Orenberg, J.B.: $\mathrm{pH}$ Profile of the adsorption of nucleotides onto montmorillonite. II. Adsorption and desorption of 5'-AMP in ironcalcium montmorillonite systems. Orig. Life Evol. Biosph. 15, 89-101 (1985) 